\title{
The application of active respiratory gating technology in lung cancer radiotherapy
}

\author{
Bo Xiao \\ Dept. Of Biomedical Engineering, Luzhou Medical College, Luzhou, Sichuan, 646000, China \\ Email address: \\ 2364061407@qq.com
}

To cite this article:

Bo Xiao. The Application of Active Respiratory Gating Technology in Lung Cancer Radiotherapy. Advances in Bioscience and Bioengineering. Vol. 2, No. 3, 2014, pp. 25-29. doi: 10.11648/j.abb.20140203.11

\begin{abstract}
Objective: The technology of $\mathrm{ABC}$ ( Active Breathing Control) was used in breath-holding time in order to improve the accuracy of radiotherapy for lung cancer patients, and the influence of respiratory movement on diaphragm motion was analyzed and evaluated, in addition the feasibility and limitation of $\mathrm{ABC}$ was also assessed. Methods: 15 lung cancer patients were selected for the training of $\mathrm{ABC}$, at the beginning of the training, let the patients practice breath-holding by themselves at home: the practice time is 30 minutes a day, one week practice, therefore the patients slowly adapted to the new breath-holding time, which is more than 40 seconds. In the CT scan and reset simulation, the images of ABC and FB( Free Breathing) were collected respectively; At the same time of precise radiation treatment, the error values between the CT images by using $\mathrm{ABC}$ system and the images of the real-time scans were collected. Results: The breath-holding time of the 15 patients were more than 40 seconds, and their average breath-holding time achieved 58.3 seconds, it is longer than that of the patients before training by 24.9 seconds. The diaphragm motion is reduced by $16.76 \mathrm{~mm}$ than that of the free breathing. In the precise radiotherapy by using $\mathrm{ABC}$, the average errors of left and right, head and feet, front and back were $0.29 \mathrm{~mm}, 0.51 \mathrm{~mm}$, $0.49 \mathrm{~mm}$; After the bed displacement, the average errors were $0.08 \mathrm{~cm}, 0.18 \mathrm{~cm}, 0.15 \mathrm{~cm}$. Conclusions: After using ABC system in the precise radiotherapy, the average error range of left and right, head and feet, front and back was within $2 \mathrm{~mm}$, and the respiratory diaphragm motion was smaller than before, so the radiation effect became more accurate, and this provided more protection for the patients.
\end{abstract}

Keywords: ABC (Active Breathing Control), Lung Cancer, CBCT (Cone Beam Computer Tomo-Graphy), Breathing Movement

\section{Introduction}

For the majority of inoperable lung cancer patients, radiotherapy is one of the main methods of treatment. But the respiratory movement is one of the factors which can cause errors in the radiotherapy. For the methods which can solve the influence of respiratory motion on radiotherapy of lung cancer, their principles are divided into two categories: one principle is the limitation of machine, that is, detectors are set to detect respiratory movement, when the respiratory motion passes over the limitative scope, the irradiation is interrupted; Another principle is the limitation of the patients, that is, by using respiratory control device, when the patient's breath reaches the set threshold, the breath is controlled for the patient's treatment. In this paper, we focused on the technology of Active Breathing Control (ABC), it is the second principle. Through the ABC training, the patient's breath was controlled, and the breath movement of the patient was kept consistent in the diagnosis and treatment, this could ensure the accuracy of radiotherapy, and the patient could receive better treatment.

\section{Methods and Materials}

\subsection{The Composition and Principle of $A B C$}

The active breathing control system, which is produced by E L E K T A company, consists of the following components: computer which is used to show the respiratory curve and process data, control module, display, mouth containing device, nose clip,sensor turbine and balloon valve which can trigger breath control, and auxiliary mirror device, etc. The patients received 30 minutes $\mathrm{ABC}$ training first, during this period, the mouth containing device was place in the patient's 
mouth, and the patient's nose was clipped with the nose clip ( but in the actual operation process, for the convenient treatment, the patient's nose was pinched by hand ), the airflow generated by the patient's breath turned the small sensor turbine in the mouth containing device. The built-in converter converts the respiratory signal into digital signal, at the same time, we can see the respiratory curves of patients on a computer screen, take the $70 \% \sim 80 \%$ of the maximal inspiratory mean value as the threshold value. In the process of CT positioning and radiotherapy, we pressed the space bar and the patient pressed the manual control switch to change the respiratory curves displayed on a computer screen from red to blue; When the respiratory threshold value was reached, the $\mathrm{ABC}$ device was activated, the balloon valve in mouth was closed, the patient's breath was obstructed, the amount of air did not change anymore, lung volume was fixed, temporarily the patient held his breath, the patient's chest cage didn't change with his breath, and this make the relative position of the surface marker and the tumor tissue fixed. Meanwhile, the interruption of the respiratory airflow make the respiration curve become a straight line, so that the patient were in the same breath phase in CT localization and treatment, and this let the patient's tumor be fixed on the same position, by using this method the radiation dose of PTV group and GTV group were improved, and the damage of normal tissue was reduced, so as to achieve the purpose of precise radiotherapy.

\subsection{Patients Selection}

From July 2013 to July 2014, 15 patients with lung cancers were selected. The selection condition: the lung functions of patients were good, their personal cognitive abilities were normal, and they agreed to the use of active breathing control system and signed information consent form, so that they can better cooperatively use this set of device.

\subsection{Respiratory Motion Measurement and the Patients Training}

Respiratory motion measurement:

The excursion degrees of the tumors in the directions of left and right $(\mathrm{X})$, front and back $(\mathrm{Y})$, head and feet $(\mathrm{Z})$ were measured for the patients with FB (Free Breathing) and with DIBH.ABC (deep inspiration breathing hold of active breathing control ), and the patients were under the simulated locator. In the later CT location, the patients accepted CT positioning image scans with the same body positions under $\mathrm{FB}$ and DIBH.ABC. The scanning range included the area from lung apex to diaphragm bottom, the two set of positioning images were transmitted into the TPS( treatment planning system, TPS) respectively. For the patient whose respiratory motion degree was smaller than $5 \mathrm{~mm}$, radiotherapy with $\mathrm{ABC}$ system was not recommended because the curative effect was not good. ( that is, ABC systems was only useful for the patient whose respiratory motion degree was greater than $5 \mathrm{~mm}$ ).

Training method for the patients: the patient nipped his nose with his left hand to make his nose not leak. the patient's right hand held the control button, a ventilation pipeline was put in his mouth. Firstly, we used the ABC system to measure the free respiratory depth of the patient, then the patient was enjoined to breathe deeply in order to measure the maximum depth of his breath, so the breath holding threshold value of ABC was obtained. The $2 / 3$ of the maximum respiratory depth was generally chosen as the threshold value. The patient needed to breath deeply, when the inspiratory depth reached the breath-holding threshold, the airbag in the $\mathrm{ABC}$ pipeline was aerated to block the pipeline, at the same time the patient started to hold his breath. If in this process the patient felt uncomfortable, he could also release the control button independently, let the airbag deflate and the pipeline become fluent. so he can recover his normal breath. The patient can exercise at home, each patient took the training of $1 \sim 2$ times / day, about 30 min / time, and he must adhere to one week training. After the patient grasped the breath-holding skill, we checked whether the breath-holding time met the requirement. Meanwhile, the patient should be encouraged in the training so as to dig out his more latent capacity, let the patient expertly master the breath-holding skill, and actively cooperate with the treatment. Finally the maximum breath-holding time and the corresponding breath-holding threshold were recorded in a notebook, and the ABC file of each patient was set up for the simulation and radiotherapy.

\subsection{Making Model of ABC}

The main process: the stereotactic body frame (SBF) was used to fix the patient, and his position was fastened by fixed bag which was made of vacuum, the device consisted of a vacuum pump and a rubber bag with plastic pellets, the bag body ws made of polyvinyl chloride(PVC) composite materials, the particles inside the bag were low density polyvinyl chloride foaming particles, which were filled with air and whose textures were soft.

The specific methods for use: a section of the connecting duct was linked to the suction inlet of the vacuum pump, the other end of the connecting duct was linked to the gas valve of the bag, and then let the patient lie his back on the locating bag, the pumping function of the vacuum pump was opened, this left a vacuum in the bag, and the bag hardened into the model.( In practice, after the vacuum bag was modelled, the patient needed to repeat again the posture, so the position became more accurate.)

The use of the vacuum bag can restrict the conscious or unconscious movement in the course of treatment, and better protect the position repeatability of every treatment, so this improved the precision of radiotherapy.

\subsection{The CT Location of $A B C$}

Firstly, the patient was supine and fixed on a vacuum pad with positioning frame, then let the patient's hands fold up and seize the holding rod on the positioning plate, make their lower limbs unbend and put together in a natural and 
comfortable posture. The fixed part of the position was from head to upper thigh. The ABC system was well connected by the staff, the mouth containing device was put in the patient's mouth, let the patient hold a hand control switch by his hand, at the same time let the patient freely breathe. The technician observed the patient's respiratory status via a notebook computer screen, and this notebook was connected with the control module. After the breath of the patient became smooth, let him take a deep breath, and the technician started the $\mathrm{ABC}$ system. When $\mathrm{CT}$ scans were performed, the images of $\mathrm{ABC}$ and $\mathrm{FB}$ were collected respectively, the general location of CT center was marked and positioned, and then through the CT location, the patient's position and the $\mathrm{X}, \mathrm{Y}, \mathrm{Z}$ coordinate parameters were strictly standardized. By using spiral CT scanner, the slice thickness was set to $3 \mathrm{~mm}$, and the lesion areas were continuously scanned in order to determine the location parts. The CT images were transmitted into the 3D planning system, the target region of radiotherapy was outlined by the oncologist, after the target region was determined, the image information was finally transmitted into the treatment planning system (TPS) so as to design the radiotherapy plan.

Notes in CT location: First of all, a large aperture CT was required in order to help the patient to put his hands above his head when he was supine, and this was convenient for scanning the patient. Secondly, the slice thickness is usually $3-5 \mathrm{~mm}$, because the line width of the location laser was greater than $1.5 \mathrm{~mm}$, if the slice thickness was too thin to generate too many CT images, the TPS could not handle the images. Finally, the time of CT scan should be as short as possible to avoid the motion effects of the patient in the process of scan. So the helical scan should be adopted as the scanning method, while the X-ray tube was rotating in scanning, the bed constantly moved forward, this technology speed up the scanning and shorten the time of scanning, from another angle the accuracy of the radiotherapy improved.

\subsection{Radiotherapy}

Before the first treatment, the verification film was shot by using EPID (Electronic Portal Imaging Device), and this played a role of verification.

CBCT (Cone Beam Computer Tomo-graphy) scan was performed daily before radiotherapy, and then the obtained images were matched with the original CT images, the position errors were achieved in the directions of front and back, head and feet, left and right. The treatment bed position was adjusted according to the error, if the value of the error was in the acceptable range, a CBCT scan was performed again to confirm the error. If every error value was smaller than $2 \mathrm{~mm}$, the patient took the treatment , and the irradiation of each field was finished by $1 \sim 2$ times of breath-holds, after the therapy had completed, the last CBCT scan was performed for verify the errors.

\section{Data Acquisition and Processing}

The errors were selected from 15 patients with lung cancer, who accepted radiotherapy everyday, and the errors were between the CBCT images and CT images ( left and right direction, head and feet direction, front and back direction). The errors were arranged in table1.

Table 1. Errors between the CBCT images and CT images in 3 directions

\begin{tabular}{llll}
\hline Standard rror & Direction of head and feet & Direction of front and back & Direction of left and right \\
\hline 1 & $0.64 \mathrm{~mm}$ & $0.21 \mathrm{~mm}$ & $0.36 \mathrm{~mm}$ \\
2 & $0.41 \mathrm{~mm}$ & $0.21 \mathrm{~mm}$ & $0.19 \mathrm{~mm}$ \\
3 & $0.28 \mathrm{~mm}$ & $0.20 \mathrm{~mm}$ & $0.10 \mathrm{~mm}$ \\
\hline
\end{tabular}

\section{Results}

The obtained average errors in the first CBCT scan were $0.29 \mathrm{~cm} 0.51 \mathrm{~cm} 0.49 \mathrm{~cm}$ respectively( direction of left and right, direction of head and feet, direction of front and back). After the bed adjustment, the average errors became $0.1 \mathrm{~cm}$ $0.18 \mathrm{~cm} 0.15 \mathrm{~cm}$ in the three directions.

Under FB, the average diaphragm motion was $18.3 \mathrm{~mm}$; Under $\mathrm{ABC}$ system, the average diaphragm motion was $1.54 \mathrm{~mm}$.

Before $\mathrm{ABC}$ system training, the average breath-holding time was 33.4 seconds; After $\mathrm{ABC}$ system training, the average breath-holding time was 58.3 seconds.

The standard error in the direction of head and feet was maximum, and the fluctuation range of the error in this direction was maximum too.

\section{Discussion}

Respiratory motion is a semi autonomous motion, which will cause large amplitude motion of thoracic and abdominal tumors. So it is necessary to compensate for tumor motion, the main methods of respiratory motion control include: passive compression technology, deep inspiration after breath hold, respiratory gating technique, real-time tracking and radiation technology[1], active breath control.

The error produced in the respiratory motion causes artifacts in the CBCT images, and this directly affects the quality of the reconstructed image [2.3]. In the process of image acquisition of the relative static target by using CBCT, the $\mathrm{kV}$, mA parameters should be selected based on the scanning object, the collected image frames should be greater than 200f, so a high quality image could be reconstructed. 
The rotation time of CBCT gantry is more than 40 seconds, so the maximum breath-holding time of the patients, who adopted $\mathrm{ABC}$, should be more than 40 seconds. After a few days of training, the average breath-holding time for every patient was more than 40 seconds, this indicated that it is feasible in practical application for the patients to let their breath-holding time be more than $40 \mathrm{~s}$, and it also plays a positive role in clinical radiotherapy. After the patient inhaled oxygen, he held his breath, and this could extend his breath-holding time and reduce the discomfort. The breath-holding threshold was $0.6 \sim 1.2 \mathrm{~L}$, the average breath-holding threshold was $0.86 \mathrm{~L}$, there was no correlation between the threshold and the breath-holding time. But the maximum breath-holding time of the patient had a close relationship with the patient's physical quality and his mental will. Therefore in the $\mathrm{ABC}$ system training, the patient needed not only the doctor's encouragement and support but also the encouragement and persistence came from the patient himself. In front of the illness, firm will is especially important.[4]

When the patient was using $\mathrm{ABC}$, in the direction of head and feet, the diaphragmatic respiratory motion of the patient was significantly less than that of the patient using free breath, this indicated that $\mathrm{ABC}$ system can primely inhibit the phrenic movement in the direction of head and feet.

When the reconstructed CBCT image was matched with the planned raw image, the error value was greatly different from the error value after the bed was moved. It showed that CBCT scan could detect the position error immediately, and CBCT scan could also control the position error in the range of $2 \mathrm{~mm}$, which was acceptable. In addition, the position error of measurement was associated with the matching methods ( bone, gray ,and manual) and the matching areas, and it had close relations with the breath-holding threshold of ABC. The lung tumors were affected by the respiratory movement, gray matching or manual matching was selected as the matching method. When the patient was using $\mathrm{ABC}$, if the fluctuation of the breath-holding threshold value was greater than $100 \mathrm{ml}$, the different values between the results obtained through matching was relatively large.[7]

\section{Conclusions}

Through the patient's daily training and oxygen intake, the $\mathrm{ABC}$ breath-holding time could be increased, at the same time, the tumor could be fixed in the breath-holding time when the patient held his breath according to the set threshold, so the quality of CBCT image could improve, correspondingly the accuracy of image matching could improve too. By means of $\mathrm{ABC}$, the tumor was fixed, the position error can be found immediately by CBCT scan, and after bed displacement, the obtained average errors were $0.1 \mathrm{~cm}, 0.18 \mathrm{~cm}, 0.15 \mathrm{~cm}$. This can control the radiotherapy precision of the lung tumor within $2 \mathrm{~mm}$, so it increased the fraction dose and total dose, finally it provided a guarantee to reduce lung injury.

ABC system was perfectly combined with the linear accelerators, three-dimensional conformal and intensity-modulated radiotherapy was performed for treating the thoracic and abdominal tumors, from the $\mathrm{CT}$ position to the process of radiotherapy, the patient's breathing movement maintain highly consistent.

This system has the following features: (1) The radiotherapy was performed only during the patient was holding his breath, and the breath-holding time for once was longer than $40 \mathrm{~s}$, and the patient generally could accept the treatment. (2) In the whole course of treatment, the amount of air of breath holding everytime was the same. (3)The patient breathed in according to the preset lung's capacity, and held his breath. (4) The irradiation volume of the normal tissue was reduced. (5) The accuracy of radiotherapy improved.

Currently, the $\mathrm{ABC}$ is mainly used in breast cancer, lung cancer and liver cancer, for which the breath has a great influence on the organ with tumor.[6] The patient accepts the left breast conserving surgery combined with radiotherapy, he is prone to the radiation sickness such as coronary heart disease, radioactive pericarditis,etc.[5]

But the $\mathrm{ABC}$ has its limits: the volume and location of the organ and target of the same patient may change to some extend in the fractional radiotherapy and in the intervals of radiotherapy. Moreover, the FRC (Functional Residual Capacity) can not be the same in every $A B C$ treatment, therefore there exists difference between the tumor locations to a certain degree. Further more, the system is complicated and it extends the time of treatment. Besides, if the patient can not firmly bite the muzzle, this will result in air leakage when the patient was holding his breath. Also, with the increase in the number of radiotherapy, radioactive inflammation and other side reactions also occur. Again, because the breath-holding time is shortened, the radiotherapy can be completed by breaking the wild into several parts, which will affect the accuracy of the radiation dose.[8]

We believe that with the further development of radiation, the $\mathrm{ABC}$ will become better, and it will be more and more used in tumor radiotherapy.

\section{References}

[1] Huang Wei, Li Baosheng. The effects and control of respiratory movement on the target area of lung tumorin the precise radiotherapy ,Journal of International Oncology,2005,1(32):53-55.

[2] Jaffray DA,Siewerdsen JH,Wang JW. Flat-panel cone beam computed tomo graphy for image-guide radition therapy.int $\mathrm{J}$ Radiati oncol Biol phys, 2002.53:1337-1349.

[3] Qnimaru R,shirato H,shimizuS .Tolerance of organs at risk in small volume,hypofractionated, image-guide radiotherapy for primary and metastatic lung cancers,int $\mathrm{j}$ Radiat oncol Biolphys,2003,56:126-135.

[4] Yao Chunping, Yu Jinming, Li Jlanbing. Application of active breathing control in postoperative radiotherapy after breast surgery,Chinese Journal of Radiation Oncology, 2006,15(3):211 214. 
[5] Qiu Jianjian, Chen Jiayi, Jiang Guoliang. The methods of breast cancer radiotherapy in which the $\mathrm{ABC}$ system was combined with forward IMRT. Chinese Journal of Radiation Oncology, 2006,15(4):304 306.

[6] Du Shisuo,Zeng Shaochong, The application study of the ABC system in the three-dimensional conformal radiotherapy of liver cancer, Chinese Journal of Radiation Oncology, 2006,15(4):368-370.
[7] Yan Jing, Yu Jinming,Application of active breathing control system in accurate radiotherapy of non-small cell lung cancer,Cancer,2006,25(10):1311-1314.

[8] Cheung PC,sixel KE.Reproducibility ofLung tumor position and reduction of Lung re\&q 8 within the planning target volume $\mathrm{u} \cdot$ sing active breathing control $(\mathrm{ABC})[\mathrm{J}]$.Int $\mathrm{J}$ Radiat Oncol BiolPhys.2003,57(5):1437 1442. 